\title{
Fractal Dimension Analysis and Statistical Processing of Paper Surface Images Towards Surface Roughness Measurement
}

\author{
Toni Kuparinen ${ }^{1}$, Oleg Rodionov ${ }^{1}$, Pekka Toivanen ${ }^{1}$, Jarno Mielikainen ${ }^{1}$, \\ Vladimir Bochko ${ }^{1}$, Ate Korkalainen ${ }^{2}$, Juha Parviainen ${ }^{2}$, and Erik Vartiainen ${ }^{2}$ \\ 1 Department of Information Technology \\ 2 Laboratory of Physics, \\ Lappeenranta University of Technology, \\ P.O. Box 20, FIN-53851 Lappeenranta, Finland \\ tkuparin@lut.fi, olerodi@hotmail.com, \\ \{ptoivane, mielikai, botchko, akorkala, jparviai, emvartia\}@lut.fi
}

\begin{abstract}
In this paper we present a method for optical paper surface roughness measurement, which overcomes the disadvantages of the traditional methods. Airflow-based roughness measurement methods and profilometer require expensive special equipment, essential laboratory conditions, are contact-based and slow and unsuitable for on-line control purposes methods. We employed an optical microscope with a built-in CCD-camera to take images of paper surface. The obtained image is considered as a texture. We applied statistical brightness measures and fractal dimension analysis for texture analysis. We have found a strong correlation between the roughness and a fractal dimension. Our method is non-contact-based, fast and is suitable for on-line control measurements in the paper industry.
\end{abstract}

\section{Introduction}

The roughness of paper surface is an important parameter in paper manufacturing and its measurement is one of central measurement problems in paper industry. The surface roughness affects the major paper property - the printing resolution, i.e. the capability to transfer without breaks and distortions the most thin printed lines, dots and their combinations. Eventually it defines the quality of a final printed product.

At present time standardized and employed in the paper industry roughness rating methods are based on measurements with the help of airflows aimed at tested surfaces. By means of specialized pneumatic devices under laboratory conditions the airflow rate between measured paper surface and a specified flat land is recorded. Such a measurement closely corresponds to the roughness of a surface, the less required the time - the rougher the surface is. The measured roughness is given in micrometers or milliliters per second according to Parker Print-Surf (PPS) and Bendtsen methods respectively [1. 
Airflow-based roughness measurement methods have certain disadvantages; central ones are a poor accuracy and incompatibility to perform measurements of fine and smooth papers. Contrary to airflow-based methods, there is a possibility to inspect a surface by means of very accurate electronic profilometer devices [2. Both described methods require expensive special equipment, essential laboratory conditions, are contact-based and slow and unsuitable for on-line control purposes methods.

Moreover, the abovementioned methods provide information on the physical roughness of a measured surface, i.e. on the microrelief and microgeometry regularity and uniformity. On the other hand, we can think of an optical roughness, that in turn refers to how paper scatters the light off the surface and appears to the observer, i.e. it characterizes optical paper properties instead. Further, we can imagine an extra roughness rating method that is intended to produce measurements of the optical paper roughness by utilization of machine vision techniques combined together with image processing.

Light transmission image analysis has been previously applied to paper formation and quality measurements 3,4,5. Studied machine vision techniques were co-occurence matrix, Fourier method and wavelets. In this research scattering light is detected and statistical image processing and fractal image analysis have been used. Fractals were first introduced in 1977 by Mandelbrot [6] and developed further in the end of 1980's by Barnsley [7. The fractal dimension is a measure of the complexity of a fractal and a review of methods can be found in [8] Kent [9] and Johansson [10] measured paper surface roughness using profilometry and studied the fractal nature of the measured profile. According to Kent 9] paper surface topography exhibits fractal characteristics. Later on, Johansson [10] also made the same conclusion using different paper grades. Contrary to the previous methods, our approach is based on an acquired microscope image and a linear dependency between the measured fractal dimension and conventional roughness has been found. Gopalakrishnan [11] applied similar fractal dimension estimation computation as we did for online monitoring of surface roughness. The difference is that they estimated the roughness of metallic surface, to which a different kind of physical reflection model has to be applied than to paper samples [12.

The article is organized as follows: in Chapter 2 we propose the method and in Chapter 3 the image processing is presented. In Chapter 4 are the experiments and in Chapter 5 the conclusions are drawn.

\section{Proposed Method}

We have developed a method based on an idea that the roughness of paper surface can be analyzed using an image formed from the light which is reflected off paper surface. Smooth papers appear to be more monotonic in terms of the reflected light spatial intensity, than rough ones that have a strong spatial variation of the reflected light intensity. 
In our method the measured surface is illuminated by grazing light. The scattering light from the sample is captured by a microscope with a built-in CCD-camera and digitized by a frame grabber. The produced digital gray-scale image is processed by a computer image processing, which provides an image measure characterizing the roughness of original surface. As in airflow methods several images of the one paper surface has to be taken and processed in order to average image measures and estimate the roughness more accurately. We have proposed statistical image processing and a fractal dimension image analysis for image processing.

\section{$3 \quad$ Image Processing}

According to the classification given in [13, we merely consider microscope paper surface images as gray-scale stochastic textures. Fig. 1 (a-c) depicts typical different roughness paper surface images.

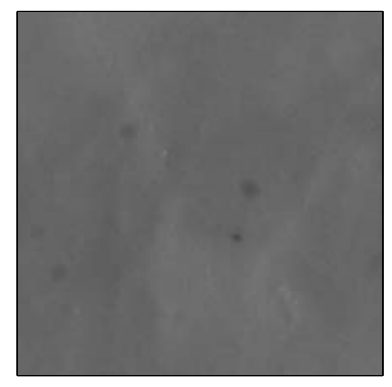

(a)

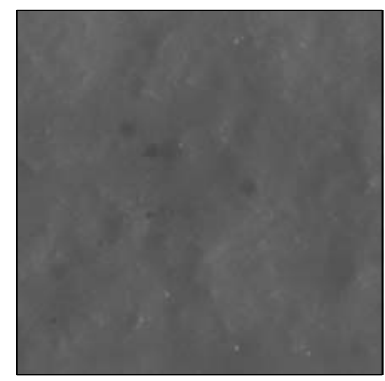

(b)

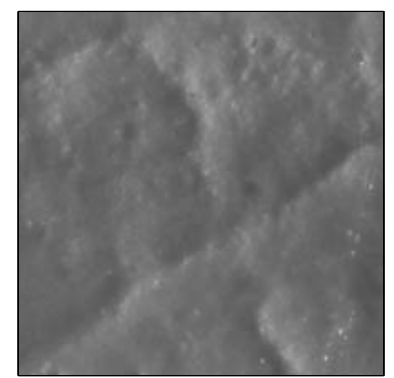

(c)

Fig. 1. Paper surface images taken by a microscope of (a) low, (b) medium and (c) high roughness

Consequently, a suitable image processing approach is statistical image processing that gives image statistical measures. Another approach is to perform a fractal dimension image analysis that estimates the complexity of image.

\subsection{Statistical Processing}

We compute a set of statistical brightness measures of a gray-scale texture image representing an inspected paper surface. The following statistical measures from the brightness histogram are computed: mean, variance, skewness, kurtosis, entropy of the brightness.

The skewness factor $s$ is a degree of symmetry, or more precisely, lack of symmetry. The kurtosis factor $k$ is a measure of whether a distribution is peaked or flat.The entropy [14] characterizes the compressibility of image and is measured 
in bits per pixel. The lower the entropy — the less the number of bits needed for coding.

The main disadvantage of previous mentioned statistical measures is that they do not take into consideration the spatial brightness distribution in the image. Therefore measures like 2D kurtosis and fractal dimension are utilized.

The 2D kurtosis is an extension of kurtosis to multidimensional data and it was introduced by Mardia [15]. Johansson [10] applied 2D kurtosis $b_{2,2}^{*}$ to gray-scale images as a measure of homogeneity of a paper surface.

\subsection{Fractal Dimension Analysis}

Microscope taken paper surface images appear as gray-scale stochastic textures that have complex spatial brightness distributions. With the help of fractal dimension analysis we have tried to overcome the abovementioned disadvantage of statistical image processing and take into account the brightness spatial distribution.

In the 1980's a discussion of fractals and chaos introduced an idea to consider texture images as fractals, 6, 7]. It leads towards a consideration of a digitized image $\boldsymbol{G}$ as a discrete set of points $\boldsymbol{S}$, each representing a particular pixel in the original image:

$$
\boldsymbol{S}=\left\{s_{i}\right\}, \quad i=1,2, \ldots, N .
$$

The fractal dimension $d$ can be computed for the set $\boldsymbol{S}$ that measures the complexity of a set. It is motivated in general by a power law relationship, if this exists, between two measures.

Let us consider a measure $N$ that is estimated by taking measurements at a scale $\epsilon$. If we change the scale $\epsilon$, most probably we get another value of the measure $N$, thus $N=N(\epsilon)$. However, it is possible to find a constant $d$ that yields an equation:

$$
N(\epsilon) \sim \frac{1}{\epsilon^{d}}
$$

The constant $d$ is the fractal dimension and not usually integer. The fractal dimension $d$ shows how rapidly the measure $N$ grows, while the scale $\epsilon$ decreases. Thereby, the fundamental idea is that the measure $N$ and scale $\epsilon$ do not vary arbitrary, but are related by a power law relationship according to Eq. 2 ,

To find the fractal dimension $d$, we solve Eq. 2 for $d$ taking a limit as $\epsilon$ approaches to zero:

$$
d=\lim _{\epsilon \rightarrow 0} \frac{\log N(\epsilon)}{\log (1 / \epsilon)} .
$$

We can try to find the fractal dimension $d$ for a given finite set $\boldsymbol{S}$ representing an image with the help of the above described approach and solving Eq. 3 . However, we will run against a problem as the limit will always yield zero, because eventually the scale $\epsilon$ will be so small that the measure $N(\epsilon)$ gets the maximum value, while $\log (1 / \epsilon)$ grows without a bound as the scale $\epsilon$ tends zero. 


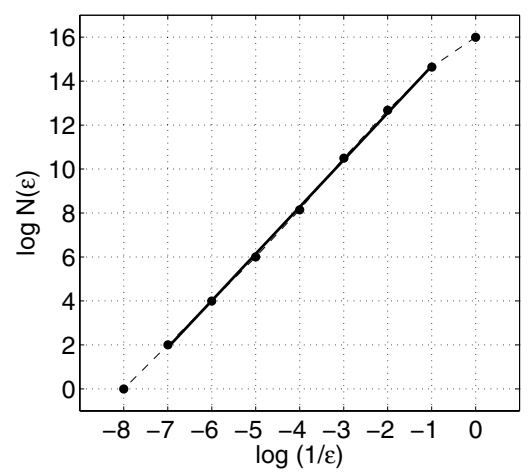

Fig. 2. Linear regression for fractal dimension estimation

In order to estimate the fractal dimension $d$ of a finite set $\boldsymbol{S}$, we must calculate values of the measure $N(\epsilon)$ for some range of the scale $\epsilon$ and take as our estimate for the fractal dimension $d$ the slope of the straight line minimizing the mean-square deviation of $\log N(\epsilon)$ vs. $\log (1 / \epsilon)$, see Fig. 2, The problem of minimization of the mean-square deviation has a solution that is given as linear regression.

The most popular and efficient way to compute the fractal dimension is through the capacity dimension by box-counting [16. The box-counting approach to compute the fractal dimension gives a systematic measurement procedure that applies to any structure in a texture plane and can be adapted for structures in multi-dimensional spaces [17. The idea of the approach is to put an image onto a regular rectangular grid of the size $\epsilon$ and simply count a number of grid cells - 'boxes' - containing some of image texture. This gives the measure $N(\epsilon)$. Then, we change the grid size $\epsilon$ to progressively smaller sizes and produce a series of measures $N(\epsilon)$. Finally, we try to fit a straight line as it is described above, the fractal dimension estimate comes as the slope of the line.

For practical purposes it is often apt to consider a sequence of grids, where the size reduces by a factor of 2 consequently 18. In such a case each cell is subdivided into four cells each of half size in the next grid. When box-counting using such grids, we arrive at a sequence of counts $N\left(2^{i}\right), i \in Z^{+}$.

In order to estimate the fractal dimension $d$ of the whole texture image a linear regression should be employed as mentioned above.

Fractal Dimension of a Gray-Level Image. We have used an extension of the box-counting approach suggested in [19. The idea of such an approach is to treat a gray-scale image $\boldsymbol{G}$ as a three-dimensional discrete set of points $\boldsymbol{S}$, where first two components $s_{0}$ and $s_{1}$ are the $x$ and $y$ coordinates of pixels, and the third component $s_{2}$ is the brightness $b$ of pixels. As a result, we deal with a $(x, y, b)$-space.

Let us consider a gray-scale image $\boldsymbol{G}$ of the size of $256 \times 256$ pixels and with 256 gray levels. Then, we can produce a three-dimensional array $\boldsymbol{S}$ of elements 
of the size of $256 \times 256 \times 256$ representing the image $\boldsymbol{G}$ : we zero all elements of $\boldsymbol{S}$ except those, whose indexes equal to the coordinates of gray-scale image pixels.

Now let us consider a three-dimensional square grid of the size $\epsilon$ of one element, see Fig 3 (a) and (b). We find a number $N(\epsilon)$ of non-zeros elements. Then, we sum the array at a time across each of three dimensions, that is equivalent of increasing the grid size $\epsilon$ up to the size of two elements, and find a new $N(\epsilon)$, see Fig 3 (c) and (d). We proceed with such a procedure, until the grid size $\epsilon$ increases up to the size of the whole array. We arrive at a sequence of grids of the sizes $\epsilon_{i}$ and corresponding measures $N\left(\epsilon_{i}\right)$, whereupon we apply the linear regression in order to compute the fractal dimension we refer to as $d$. We have to mention that ending points should be dropped due to a phenomenon described in 18. See Fig. 2 for the illustration. The fractal dimension $d$ characterizes the complexity of the texture of a gray-scale image.

\section{Experimental Setup and Results}

We have implemented all necessary computational algorithms for statistical image processing and image fractal dimension analysis in Matlab and run all experiments under the Matlab environment.

In our set of experiments we have used three test sets of paper samples. In every set we have produced 10 gray-scale microscope images of each paper specimen. Each image is averaged from 5 images from the same area to reduce white noise. The size of images was 512 x 512 pixels with 8 bits per pixel and a image shows an area of size $0.3 \mathrm{~mm} \times 0.3 \mathrm{~mm}$ of the paper surface. For each test set illumination was adjusted according to paper brightness and roughness and then held constant during the measurement of a set.

The results from the first test set are reported in more detail to illustrate the method. The reported values for paper specimens are the average values of a measurement from 10 images. From all the test sets the correlation of roughness and each measure are reported.

In the first set, denoted as UPM A, there are 6 coated, calendered paper specimens of the different roughness measured by PPS. The roughness of paper specimens appear to possess values of $0.63,0.66,0.67,0.71,0.91$ and $0.95 \mu \mathrm{m}$.

In the second set, denoted as UPM B, there are 6 coated, calendered samples of two different paper grades measured by PPS. The first three samples are for gravure printing and the roughness of paper specimens are $0.62,0.67,0.70 \mu \mathrm{m}$. The last three samples are for offset printing with the roughness values 0.99 , $1.08,1.08 \mu \mathrm{m}$.

In the third set, denoted as Stora Enso, there are 2 samples of base board, a sample of coated board, a sample of uncoated paper and a sample of coated paper measured by Bendtsen. The roughness of base boards are 303 and $447 \mathrm{ml} / \mathrm{min}$, coated board $11 \mathrm{ml} / \mathrm{min}$, uncoated paper $228 \mathrm{ml} / \mathrm{min}$ and coated paper $8 \mathrm{ml} / \mathrm{min}$. 


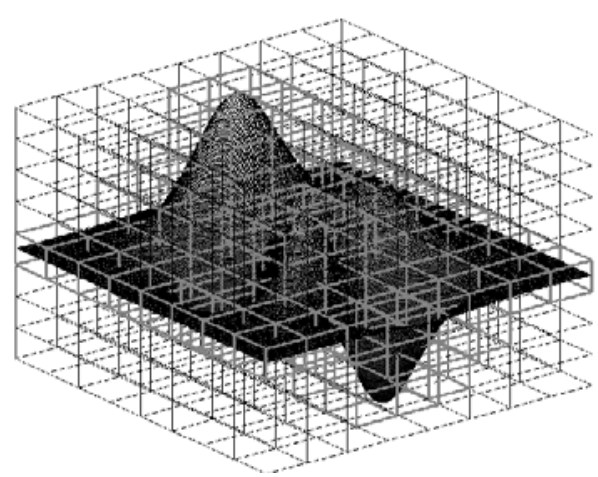

(a)

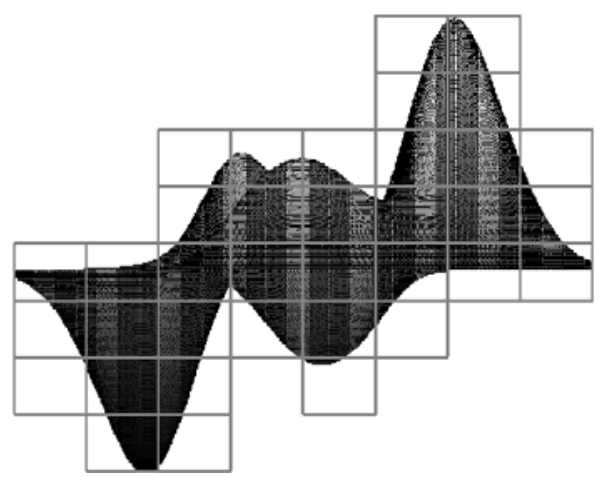

(c)

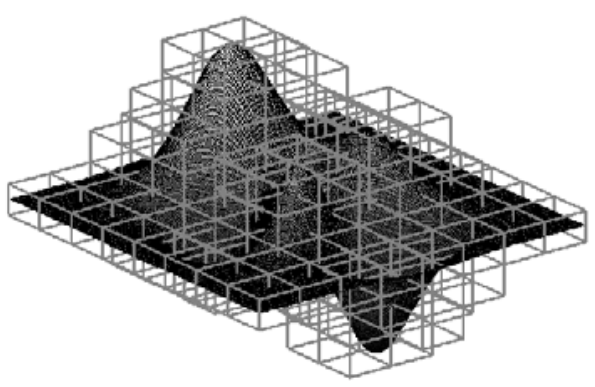

(b)

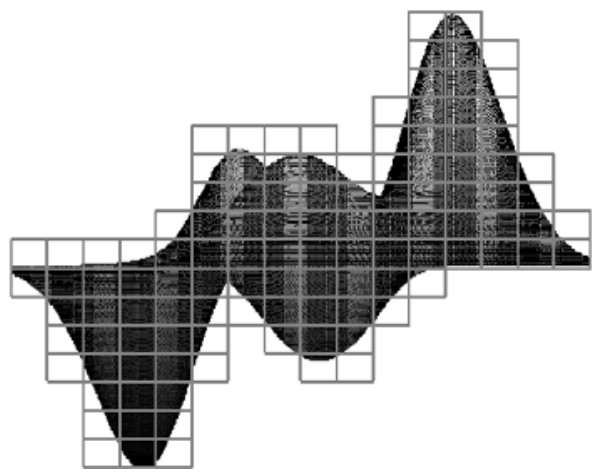

(d)

Fig. 3. 3D Box-counting algorithm: (a) regular rectangular grid over the image, (b) grid cells containing some image texture (denoted as gray boxes), (c-d) 2D presentation of different grid sizes $\epsilon$.

\subsection{Results from Statistical Processing}

We have computed statistical measures of our test set of images and the correlation with the physical roughness. See Table 1 for image statistics averaged for each paper specimen of UPM A test set.

We have found out that some of statistical measures show the strong correlation with the roughness for some test sets, see Table 2. For instance the brightness mean has a high correlation 0.98 and 0.91 in test sets UPM A and UPM B, respectively. Unfortunately, in test set Stora Enso the correlation is only 0.73 . The average correlation of the three test sets for the brightness mean, variance and $2 \mathrm{D}$ kurtosis were $0.87,0.87$ and 0.88 , respectively. These three were the best statistical measures. 
Table 1. Statistical measures: mean $\mu$, variance $\sigma^{2}$, skewness $s$, kurtosis $k$, entropy $\eta$ and 2D kurtosis $b_{2,2}^{*}$ and fractal dimension $d$

\begin{tabular}{|c|ccccccc|}
\hline$r, \mu \mathrm{m}$ & $\mu$ & $\sigma^{2}$ & $s$ & $k$ & $\eta$ & $b_{2,2}^{*}$ & $d$ \\
\hline 0.63 & 98.22 & 14.910 & 0.901 & 14.33 & 3.929 & 347.3 & 2.163 \\
0.66 & 98.61 & 16.170 & 0.619 & 9.880 & 3.998 & 348.1 & 2.165 \\
0.67 & 98.59 & 18.080 & 0.915 & 12.29 & 4.051 & 345.9 & 2.164 \\
0.71 & 98.09 & 16.850 & 0.847 & 9.289 & 4.016 & 347.3 & 2.159 \\
0.91 & 99.79 & 25.410 & 1.892 & 28.15 & 4.253 & 354.9 & 2.125 \\
0.95 & 101.9 & 29.080 & 1.674 & 16.19 & 4.316 & 359.2 & 2.125 \\
\hline
\end{tabular}

Table 2. Correlation between different image measures and the roughness

\begin{tabular}{|c|ccc|}
\hline Measure & UPM A & UPM B & Stora Enso \\
\hline$\mu$ & 0.98 & 0.91 & 0.73 \\
$\sigma$ & 0.94 & 0.84 & 0.83 \\
$s$ & 0.69 & 0.77 & 0.81 \\
$k$ & 0.98 & 0.49 & 0.66 \\
$\eta$ & 0.98 & 0.56 & 0.81 \\
$b_{2,2}^{*}$ & 0.96 & 0.90 & 0.77 \\
$d$ & -0.99 & -0.96 & -0.91 \\
\hline
\end{tabular}

\subsection{Results from Fractal Dimension Analysis}

We have computed fractal dimension $d$ explained above for test sets. See the last column of Table 1 for numerical results of UPM A test set. We have revealed that microscope paper surface images are fractal: a logarithmic scale plot of boxcounting perfectly fits a straight line, see Fig. 2 for an example fit of a paper surface image from UPM A test set.

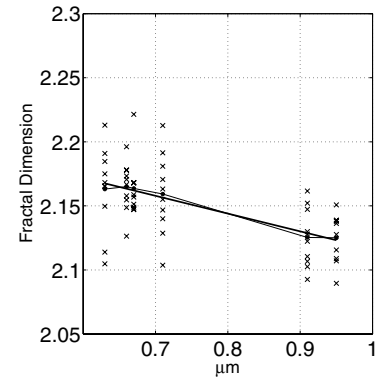

(a)

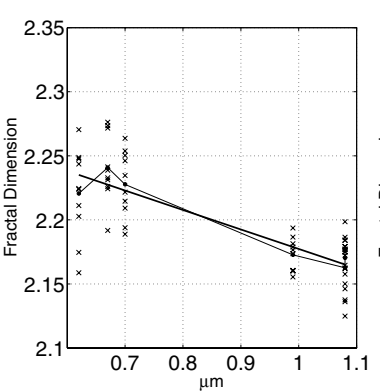

(b)

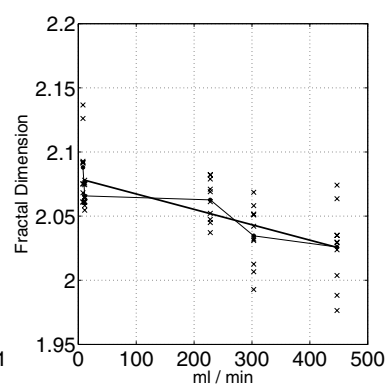

(c)

Fig. 4. (a-c) Fractal dimension $d$ vs. the roughness (curved lines) and least-square lines (straight lines) of test sets UPM A, UPM B and Stora Enso, respectively 
The fractal dimension computation has turned out that it can be efficiently used in order to estimate the surface roughness and leads to the high correlation with the latter. See Table 2 for the obtained correlation values and plots in Fig. $4(\mathrm{a}-\mathrm{c})$ of fractal dimension $d$. In a case of fractal dimension $d$ we have received in average the highest correlation, -0.95 , in our set of experiments. The average correlation is significally higher for fractal dimension than for statistical measures.

In Fig. 4 ( $\mathrm{a}-\mathrm{c})$ the variance of fractal dimension measurements seems relatively high due to scaling of the vertical axis. However, the variance of 10 fractal dimension and PPS measurements are similar; the variance divided by the mean of measurements is 0.02 and 0.04 , respectively. Therefore, fractal dimension may be successfully used in the paper surface roughness estimation. However, the current method requires calibration of measurement device for each paper type.

\section{Conclusions}

We have studied the possibility to utilize machine vision techniques in order to perform measurements of the roughness of paper surface. We have used images taken by a microscope with a built-in CCD-camera and employed statistical image processing and image fractal dimension analysis.

We have found that the proposed method has a great potential and performs well compared to standardized and industrially employed airflow-based methods. The proposed method overcomes the disadvantages of existing methods, namely the need for expensive special pneumatic equipment, essential laboratory conditions and time consumption to carry out measurements. Our method is also non-contact-based, fast and is suitable for on-line control measurements in the paper industry.

The obtained results are promising and indicate that the employed image taking and processing can successfully be used in order to measure the paper surface roughness. We have found a set of image measures, which show strong correlation with the roughness of original surface.

We have studied the correlation between the roughness and image statistics. We found out that the image brightness variance, mean and $2 \mathrm{D}$ kurtosis performs reasonably well for some test sets, but unfortunately not for all.

The best results and correlation have been achieved by applying fractal dimension image analysis. We have investigated that microscope paper surface images are fractal and image fractal dimension depends on the roughness of the original surface and show high correlation values for all applied test sets.

In our experiments we have used and worked with uncoated and coated, calendered paper samples and cardboards and the suitability of our method for even more diverse types of papers and cardboards is under research. In the future the method will be developed so that, there will be no need for a calibration of measurement device for each paper type. The ultimate goal is to perform the measurements online on a paper machine. 


\section{Acknowledgements}

The authors gratefully appreciate the provided funding from European Regional Development Fund (ERDF) and National Technology Agency of Finland (TEKES). Special thanks come to the Laboratory of Physics of Lappeenranta University of Technology, Finland for the help in microscope image acquisition and UPM and Stora Enso for providing paper samples.

\section{References}

1. ISO 8791/1-1986 (E): (Paper and board - determination of roughness and smoothness ( air leak methods). part 1: General method)

2. Wagberg, P., Johansson, P.: Surface profilometry - a comparison between optical and mechanical sensing on printing papers. Tappi Journal 76 (1993) 15-121

3. Cresson, T., Luner, P.: The characterization of paper formation. part 2: The texture analysis of paper formation. Tappi 73 (1990) 175-184

4. Bernie, J., Douglas, W.: Local grammage distribution and formation of paper by light transmission image analysis. Tappi 79 (1996) 193-202

5. Bouydain, M., Colom, J., J., P.: Using wavelets to determine paper formation by light transmission image analysis. Tappi 82 (1999) 153-158

6. Mandelbrot, B.: The Fractal Geometry of Nature. Freeman and Company (1977)

7. Barnsley, M.: Fractals Everywhere. Academic Press Inc. (1988)

8. Bisoi, A.K., Mishra, J.: On calculation of fractal dimension of images. Pattern Recognition Letters 22 (2001) 631-637

9. Kent, H.J.: The fractal dimension of paper surface topography. In: TAPPI/CPPA International Printing and Graphics Arts Conference, Vancouver, Canada (1990) 73-78

10. Johansson, J.O.: Models of Surface Roughness with Applications in Paper Industry. $\mathrm{PhD}$ thesis, Lund Institute of Technology (2002)

11. Gopalakrishnan, S.: Development of a prototype system for on-line monitoring of surface roughness using fractal geometry. Master's thesis, Institute of System Research (1994)

12. Klinker, G.J.: A physical approach to color image understanding. A K Peters, Wellesley, Massachusetts (1993)

13. Visa, A.: Texture Classification and Segmentation Based on Neural Network Methods. PhD thesis, Helsinki University of Technology (1999)

14. Shannon, C.E.: A mathematical theory of communication. Bell System Technical Journal vol. 27 (1948) 79-423,623-656

15. Mardia, K.V., Kent, J.T., Bibby, J.M.: Multivariate Analysis. 2nd edn. Academic Press, London, UK (1980)

16. Georgilli, A., et al.: An efficient procedure to compute fractal dimensions by box counting. Physical Letters A 115(5) (1986) 202-206

17. Peitgen, H., et. al: Chaos and Fractals: New Frontier of Science. Springer-Verlag (1992)

18. Liebovitch, L.S., Toth, T.: A fast algorithm to determine fractal dimensions by box counting. Physical Letters A 141(8,9) (1989) 386-390

19. Gagnepain, J., RoQues-Carmes, C.: Fractal approach to two-dimensional and three-dimensional surface roughness. Wear 109 (1986) 119-126 